\title{
Improved Routing Protocol in Mobile Ad Hoc Networks Using Fuzzy Logic
}

\author{
Seyed Amin Hosseini Seno, Ali Abdi Seyedkolaei* \\ Ferdowsi University of Mashhad \\ Faculty of Engineering, Department of Computer Engineering \\ *Corresponding author, e-mail: aabdi64@gmail.com
}

\begin{abstract}
In mobile ad hoc networks, route selection is one of the most important issues that is studied in these networks as a field of research. Many articles trying to provide solutions to choose the best path in which the important parameters such as power consumption, bandwidth and mobility are used. In this article, in order to improve the solutions presented in recent papers parameters such as power remaining, mobility, degree node and available bandwidth are used by taking the factors for each parameter in proportion to its influence in choosing the best path. Finally, we compare the proposed solution with the three protocols IAOMDV-F, AODVFART and FLM-AODV with the help of OPNET simulation program based on network throughput, routing discovery time, the average number of hops per route, network delay.
\end{abstract}

Keywords: fuzzy logic, mobile ad-hoc network, routing, efficiency

Copyright $\odot 2018$ Universitas Ahmad Dahlan. All rights reserved.

\section{Introduction}

In recent decades, following the advent of wireless communications technology, new communication networks such as contingency networks have been introduced that have had important applications in the military, industrial and social spheres [1]. Mobile ad hoc networks, independent set of mobile hosts that wireless channels with limited bandwidth with each other. The topology and arrangement of these types of networks are rapidly and unpredictably changed due to the mobility of the nodes. Due to the easy expansion of the network, some mobile ad hoc networking applications include use in emergency and rescue operations, conferences, implementation of networks between cars and personal area networks. Given the nature of these networks, each node in such networks is not only responsible for discovering a new path for data transfer from the source node to the destination node, but also as a router for transmitting messages [2].

There are no central controllers in these networks. A routing operation that involves recognizing the network topology and delivering messages are distributed in a distributed and independent way by nodes in the network, which can act as routers. In a mobile ad hoc network, a message sent by a node to the entire neighboring node which is located in the radius of the transfer. The problem of coating and binding has a great influence on the efficiency of the mobile contingency network [3]. The mobility of nodes and the possibility of momentary variations in network topology, variety of equipment, energy and bandwidth constraints, Search and maintaining an optimal path in the network to other networks is a major challenge [4].

In mobile ad hoc networks, route selection is one of the most important issues that as a field of research are studied in these networks. Mobility nodes in mobile ad hoc networks are the biggest challenge. Because that makes links and routes destroyed and topology dynamically change that this changes difficult network management. In general, high mobility changes the topology frequently, which reduces the network performance. It is difficult to choose a sustainable path to get better performance, because less time is spent on maintenance of paths and more time is available to send useful information. This interaction between mobility, topology and performance in the modeling of mobile ad hoc networks is fundamental and rarely analyzed. Many articles trying to provide solutions to choose the best path in which the important parameters such as power consumption, bandwidth and mobility is used. In this article 
in order to improve the solutions presented in recent papers [5-7] parameters such as power remaining, mobility, degree node and available bandwidth are used by taking the factors for each parameter in proportion to its influence in choosing the best path. The values of these parameters are defined as linguistic for input fuzzy system and we are considering fuzzy rules stated obtains the output value of link stability. Fuzzy logic is a kind of computer science approach that uses correct grade in Boolean logic rather than the common or false (zero or one) commonly used modern computer system. Consider fuzzy logic 0 and 1 as reality states, but there are several correct states between these two states. For example, the result of a comparison between two things in height may be neither short nor long, but 0.38 higher than one. Fuzzy Logic, the provider of the framework for controlling systems in which exact numerical information from the system is not needed and the exact process identification mechanism is not required. Fuzzy logic can analyze phenomena that are not repeatable, or they cannot be regarded as an experience and under varying conditions under different circumstances [8]. Fuzzy logic is an important tool for designing controllers for nonlinear systems. The reason for using fuzzy logic in ad hoc networks is the uncertainty in the values of the parameters that influence the choice of optimal path, especially for mobile contingency networks. A fuzzy logic system is a system that provides a natural way to accept multiple inputs of constraints that have ambiguous values in nature.

There are two types of fuzzy logic inference system, one of which is Mamdani type, and another is Sugeno type. Mamdani system is more popular and more used. The function of both Mamdani and Sugeno systems is similar. But the difference is that in the Mamdani system, the fuzzy output is neither linear nor fixed. But in the Sugeno system, the output is fuzzy or linear or fixed. In this paper, the Mamdani system is used for the fuzzy logic system, since its fuzzy output is linear and not constant.

Another point in this article is that the factor of motility parameter is considered negative, since the mobility of nodes causes the changing network topology. This change leads to destroy the best path and therefore do again which will be a large overhead for network route discovery process that will be in terms of energy consumption.

Next sections will be discussed in the rest of the paper including: in section 2, we describe related work. In Section 3, we present our proposed model. In Section 4, we present simulation results of IAOMDV- F, AODVFART and FLM-AODV protocols and our proposed model and we will do a comparison based on their results. Finally, in section 5 , we will do conclusion based on simulation results and comparison between these three protocols.

\section{Related Work}

DFES-AODV [9] Routing Protocol is one of the reactive protocol in which it is used fuzzy logic and reinforcement learning. In this protocol, it is used on the dynamic membership function to enhance the adaptive of legacy fuzzy logic systems. Using dynamic fuzzy logic for energy aware routing in mobile ad-hoc networks is more appropriate than routing protocol based on reinforcement learning. In the route discovery phase of the DFES-AODV protocol, each node performs its route requests based on Mamdani fuzzy logic system that in fuzzy logic system is used parameters power remaining and energy consumption by each node that the amount of power remaining in this protocol is dynamically in contrast to previous protocols.

Abbaset et al. in [10] proposed reactive routing protocol based on fuzzy logic to increase performance AODV routing protocol by selecting the most trusted nodes to create the best route from the source node to the destination node. In this protocol, fuzzy logic system is used as parameters such as power remaining, node mobility, and number of fop counts to evaluate each node to select high-priority trusted nodes to create the best path. Simulation results show that the proposed protocol performs better than AODV protocol and in terms of average control overhead, packet delivery ratio, network throughput and average end-to-end delay is more suitable than AODV protocol.

Prasanthi and et al. in [11] using fuzzy logic in AODV routing protocol, reduce delays and congestion in AODV protocol and resulting in improved network performance than AODV protocol. They consider the number of hop counts and network size as input parameters of fuzzy logic system in the proposed method. The simulation results using software OPNET showed that the average end to end delay and packet delivery ratio is improved compared to AODV protocol. 
Baskaran et al [12] Improved Bee routing algorithms using fuzzy logic. Their goal presenting this algorithm is to improve the reliability and limited bandwidth in routing algorithms. In their research, they have used ideas of bee colony algorithm for finding the shortest path between two source and destination nodes using the least number of steps. They used fuzzy logic with power nodes, bandwidth and data traffic to select the best path. Their proposed algorithm with AODV and Bee-MANET algorithms has been compared in terms of quality of service, delay characteristics and stability path.

FBORP protocol [13] was recommended to improve the original AODV routing protocol in mobile ad hoc networks to increase the stability using fuzzy logic. In this protocol have been used three important parameters such as energy, bandwidth and node mobility. The simulation results showed that this protocol compared with the original AODV protocol has better routing discovery time, the average packet overhead, the average throughput and average final delay.

AODVFART Protocol [5] analyzes the performance of AODV protocol with optimized active route timeout interval using fuzzy logic system. In this protocol, fuzzy logic system has been used to decide on the amount of the active route timeout that its input parameter of fuzzy logic system is transmission power and mobility of the nodes. The simulation results showed that performance of AODVFART protocol is better than AODV protocol in terms of average throughput, average end-to-end delay and average jitter.

IAOMDV-F Protocol [6] which is improved of AODV protocol has used fuzzy logic system to select the best path. Fuzzy logic system used in this protocol chooses the best path among multiple paths available to optimize energy consumption and the stability path. In this protocol, fuzzy logic system uses three input parameters, average mobility, queue length and the distance between nodes to select the best path. The simulation results showed that performance of IAOMDV-F protocol compared to AODV, AOMDV and AOMDV-F protocols is better in terms of end-to-end delay, throughput, and packet delivery ratio.

FLM-AODV Protocol [7] uses fuzzy logic to improve the AODV protocol. In the fuzzy system, two parameters are considered the hop count and mobility. These two parameters cause a better path than the AODV protocol. The simulation results show that the FLM-AODV protocol improves average end-to-end delay and packet delivery ratio compared to the AODV protocol.

\section{Proposed Fuzzy-Based Model}

In mobile ad hoc networks, route selection is one of the most important issues that is studied as a field of research in these networks. Therefor their stability is dependent upon the action of nodes in particular moving nodes. In mobile ad-hoc network, high mobility of nodes is one of the main reasons for links failure; because it causes links and routes to be disappeared then topology dynamically changes that this change makes it difficult to manage the network. Generally, high mobility and topology frequently change that these changes will reduce the performance of mobile ad hoc networks.

Many protocols has been introduced to choose the best path for sending data from the source node to the destination node in which the parameters such as power consumption, bandwidth and mobility are used to choose the best path. One of the most popular routing protocols in mobile ad-hoc network is AODV protocol where paths are discovered only when required and used during the time of their maintenance. Many methods to improve AODV protocol have been presented in numerous articles. The most recent of these methods are IAOMDV-F, AODVFART and FLM-AODV protocols. In AODVFART the protocol from transmission power and nodes mobility parameters and in IAOMDV-F the protocol from queue length and the distance between nodes parameters is used to choose the best path from the source node to the destination node. In FLM-AODV the protocol from hop count and nodes mobility parameters is used to choose the best path. In this paper, it has been tried that in addition to parameters of previous papers from other parameters such as the degree node, bandwidth and energy to be used. The degree node parameter causes to select the nodes close together as the route node. This will lead to network outages occur later and we have energy and less overhead to create the path. Also available bandwidth parameter causes that among the routes available we select routes that have more stability than the other routes. And also discuss the quality of service, reduce end-to-end delays and reduce packet delivery ratio which is taken into account. Energy parameter also causes the nodes that are selected to create a

TELKOMNIKA Vol. 16, No. 2, April $2018: 728-738$ 
route including a high lifetime to cause disconnected communication route. In the following, in different subsections, parameters used in fuzzy logic will be introduced along with the concept "fuzzy rules and their membership functions".

\subsection{The Effect of Energy Nodes on the Link Stability}

Energy node is an evaluation criterion for link stability. The power remaining can be used as a parameter to describe the energy situation i used as a custom node. We denote the power remaining as. The value of is obtained with a very simple way with the help of Equation (1):

$$
p_{\text {remi }}=p_{a v}-p_{r t}
$$

Where, is the available energy of node is the required transmit power of a data message of bbits between two nodes by a transmission range of $d$ meters. The value of is obtained with the help of Equation (2):

$$
p_{r t}(b, d)=p_{\text {elec }} * b+\left(e_{f s} * b * d^{2}\right)
$$

Where, $e_{f s}=10 \mathrm{PJ} / \mathrm{bit} / \mathrm{m} 2$ is the energy consumed in free space at the output transmitter antenna for transmitting one meter and $p_{\text {elec }}=50 \mathrm{~nJ} / \mathrm{bit}$ is the energy expended in the radio electronics [13-14].

The lifetime of the route has relationship with the minimum $p_{\text {remi }}$ for all nodes in the path. That is, if the energy of any node in the path is exhausted, then the link would crash. Thus, $p_{\text {remi }}$ represents the power remaining of the node $\mathrm{i}$, therefore, the lifetime of the route is related to $\min p_{\text {remi }}$, that is:

$$
\text { power }=\min p_{\text {remi }} \quad \forall \text { node } \rightarrow i \in \text { path }
$$

If the power is low, the probability of the link broken will be high. Thus, the rules for the relationship between power and link stability should be as follows:

a. P1: If power is high then link stability must be high.

b. P2: If power is medium then link stability must be medium.

c. P3: If power is low then link stability must be low.

\subsection{The Effect of Bandwidth on the Link Stability}

We use the available bandwidth between two adjacent nodes, to describe the bandwidth condition. A simple method gives the available bandwidth based on transmission speed and can be used to measure the bandwidth for an (I, J) link. This link has an available bandwidth that is obtained with the help of Equation (4):

$$
\text { Available_bandwith }(I, J)=(1-u) * \text { Bandwith }(I, J)
$$

Where $u$ is the link utilization $(u=A(t) / t ; A(t)$ is the total amount of time in which the link is used by nodes during an interval of time t). The link stability has relationship with the minimum available bandwidth for all nodes in the path, when the route is congested, the shared bandwidth of channels would be reduced, so that the route became unstable due to the retransmission of the lost packets. That is, if the available bandwidth of any node in the path is reduced, then the path would crash, therefore, the lifetime of the route is related to min available bandwidth, for all nodes in the path. If the available bandwidth is low, the probability of the link broken will be high. The rules for the relationship between available bandwidth and link stability are

a. B1: If available bandwidth is high then link stability must be high.

b. B2: If available bandwidth is low then link stability must be low

Improved Routing Protocol in Mobile Ad Hoc Networks Using... (Seyed Amin Hosseini Seno) 


\subsection{The effect of node mobility on the link stability}

MANETs experience dynamic changes in network topology because of the unrestricted mobility of the nodes in the network. If the end nodes experience much movement, then it is highly probable that their path will break. The node movement can be measured by the number of sent control packets between two sampling intervals. Sent control packet is any message of the following type: RREQ, RREP, RERR and RREP_ACK.

If the RREP packet records the number of sending control packet of the intermediate nodes is high, it represents the route is probably unstable. So, high number of these packets means high probability to loose some of its current links or packets [15]. In general, a rule can be defined: When sent control packet is high, the link stability must be low and vice versa. Consequently the following fuzzy rules are proposed:

a. M1: If sent control packet is high then link stability must be low.

b. M2: If sent control packet is medium then link stability must be medium.

c. M3: If sent control packet is low then link stability must be high. In general, the link stability is an inverse ratio sent control packet.

\subsection{The effect of node degree on the link stability}

Degree of a node ( $\mathrm{Di}$ ) is defined as the number of neighbouring nodes. To compute node degree the concerned device broadcast a HELLO packet. The nearby nodes that can hear any HELLO packet record the source node's address as its neighbour node. Then the code can compute total number of neighbours by counting the number of HELLO packets that it hears. A table of nodes is maintained by each node to use it in future communication. Thus, if the degree of any node in the path is increased, then the path would stable, therefore, the lifetime of the route is related to max node degree, for all nodes in the path.

If the node degree is low, the probability of the link broken will be high. Thus, the rules for the relationship between node degree and link stability should be as follows:

a. D1: If node degree is high then link stability must be high.

b. D2: If node degree is low then link stability must be low.

Now according to the defining the rules of four parameters effective in stability of link, it can be specified as the table of fuzzy rules base to determine priority selection of the next node in the routing as shown in Table 1.

Table 1. Fuzzy Logic Rules Base

\begin{tabular}{|c|c|c|c|c|c|}
\hline \multirow[b]{2}{*}{ No. i } & \multicolumn{4}{|c|}{ Input } & \multirow{2}{*}{$\begin{array}{c}\text { Output } \\
\text { Link Stability }\end{array}$} \\
\hline & $\begin{array}{l}\text { Available } \\
\text { Bandwidth }\end{array}$ & $\begin{array}{c}\text { Power } \\
\text { Remaining }\end{array}$ & $\begin{array}{c}\text { Node } \\
\text { Degree }\end{array}$ & $\begin{array}{c}\text { Sent Control } \\
\text { Packet }\end{array}$ & \\
\hline 1 & Low & Low & Low & Low & Very Low \\
\hline 2 & Low & Low & Low & Medium & Very Low \\
\hline 3 & Low & Low & Low & High & Very Low \\
\hline 4 & Low & Low & High & Low & Low \\
\hline 5 & Low & Low & High & Medium & Rather Low \\
\hline 6 & Low & Low & High & High & Rather Low \\
\hline 7 & Low & Medium & Low & Low & Low \\
\hline 8 & Low & Medium & Low & Medium & Rather Low \\
\hline 9 & Low & Medium & Low & High & Rather Low \\
\hline 10 & Low & Medium & High & Low & Medium \\
\hline 11 & Low & Medium & High & Medium & Medium \\
\hline 12 & Low & Medium & High & High & Medium \\
\hline 13 & Low & High & Low & Low & Low \\
\hline 14 & Low & High & Low & Medium & Rather Low \\
\hline 15 & Low & High & Low & High & Rather Low \\
\hline 16 & Low & High & High & Low & Medium \\
\hline 17 & Low & High & High & Medium & High \\
\hline 18 & Low & High & High & High & High \\
\hline 19 & High & Low & Low & Low & Very Low \\
\hline 20 & High & Low & Low & Medium & Low \\
\hline 21 & High & Low & Low & High & Low \\
\hline 22 & High & Low & High & Low & Rather Low \\
\hline 23 & High & Low & High & Medium & Rather Low \\
\hline 24 & High & Low & High & High & Rather Low \\
\hline 25 & High & Medium & Low & Low & Medium \\
\hline
\end{tabular}


Table 1. Fuzzy Logic Rules Base

\begin{tabular}{cccccc}
\hline No. i & $\begin{array}{c}\text { Available } \\
\text { Bandwidth }\end{array}$ & $\begin{array}{c}\text { Power } \\
\text { Remaining }\end{array}$ & $\begin{array}{c}\text { Node } \\
\text { Degree }\end{array}$ & $\begin{array}{c}\text { Sent Control } \\
\text { Packet }\end{array}$ & $\begin{array}{c}\text { Output } \\
\text { Link Stability }\end{array}$ \\
\hline 26 & High & Medium & Low & Medium & Medium \\
27 & High & Medium & Low & High & Medium \\
28 & High & Medium & High & Low & High \\
29 & High & Medium & High & Medium & High \\
30 & High & Medium & High & High & Very High \\
31 & High & High & Low & Low & Medium \\
32 & High & High & Low & Medium & High \\
33 & High & High & Low & High & High \\
34 & High & High & High & Low & High \\
35 & High & High & High & Medium & Very High \\
36 & High & High & High & High & Very High \\
\hline
\end{tabular}

In the following, membership functions of four input parameters, sent control packet, power remaining, available bandwidth and degree node and output parameter of link stability, respectively will be shown in Figures 1 and 2 .
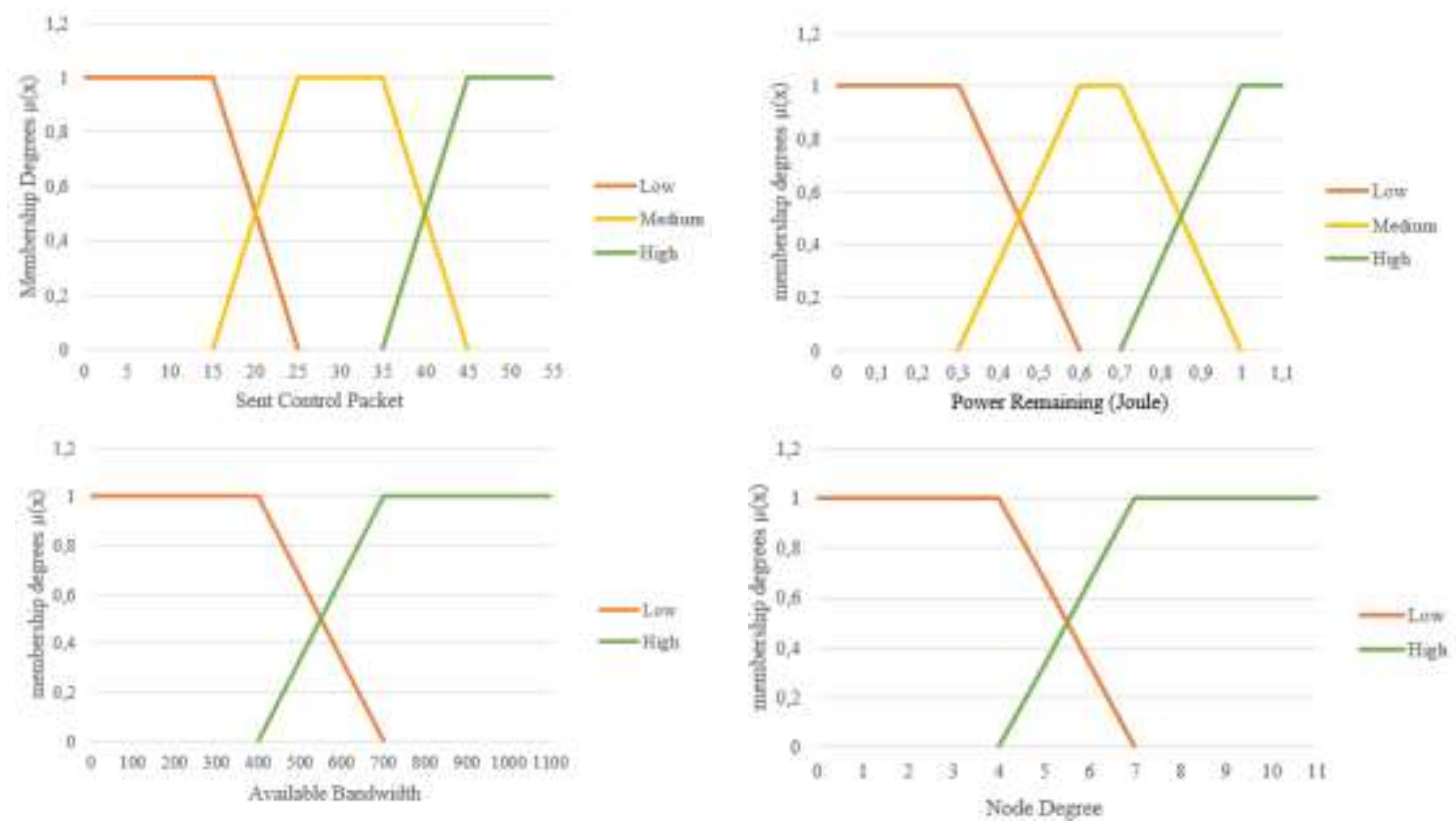

Figure 1. Membership function for sent control packet, power remaining, available bandwidth and node degree respectively

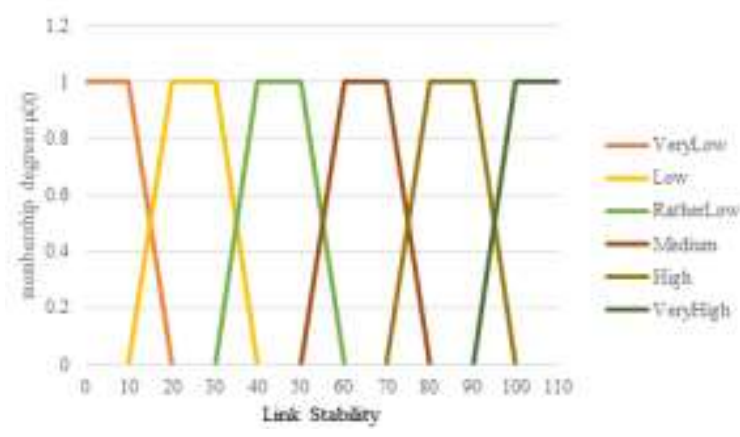

Figure 2. Membership function for link stability 
Overally, we can use the base rules expressed in the Table 1 to use in the fuzzy systems in the following format:

If available bandwidth is $\mathrm{Ai} 1$ and power remaining is $\mathrm{Ai} 2$ and sent control packet

is $\mathrm{Ai} 3$ and node degree is $\mathrm{Ai} 4$ then Stable Path is $\mathrm{Bi}$

Where Ai2, Ai3 are the linguistic labels low, medium and high and Ai1, Ai4 are the linguistic labels low and high and $\mathrm{Bi}$ are the linguistic labels very low, low, rather low, medium, high and very high of the ith rule.

Based on the defined base rules according to with Equation (5), the link stability value for different routes to the same destination can be calculated using Equation (6). In this equation $\mathrm{SP}(\mathrm{i}), \mathrm{B}(\mathrm{i}), \mathrm{M}(\mathrm{i}), \mathrm{D}(\mathrm{i})$ and $\mathrm{P}(\mathrm{i})$ is the membership function related to the link stability, available bandwidth, sent control packet, degree node and power remaining, respectively.

$$
S P(i)=\alpha_{i} * B(i)-\beta_{i} * M(i)+\gamma_{i} * D(i)+\delta_{i} * P(i)
$$

In Equation (6) it is considered four-parameter available bandwidth, sent control packet, degree nodes and power remaining with various factors that these factors can be in accordance with system requirements and have a significant impact on the link stability. Considered factors to any parameters take values between 0 and 1 according to the priority of each parameter that the range of the values shown in Figure 3 . The priority of parameters is from top to bottom in the order which includes power remaining, degree node, available bandwidth and sent control packet.

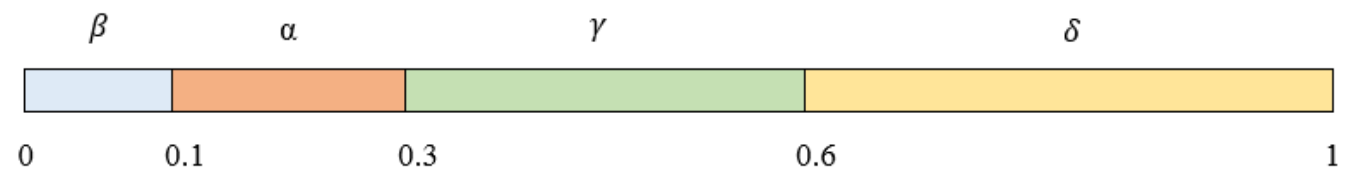

Figure 3. The range of values for parameter factors

The important point is the link stability which has an inverse ratio corresponding to sent control packet and direct ratio to the available bandwidth, node degree and power remaining. As a result, value of link stability i.e. SP(i) will be obtained through the combination of these parameters.

\section{Simulation Results}

In this section, we compare the performance of the proposed model with the IAOMDVF, AODVFART and FLM-AODV protocols using OPNET simulation software. we compared the 20 nodes with the assumptions described in Table 2 and obtain index values include network throughput, routing discovery time, the number of hops, network delay, package delivery fraction and number of packets loss from the simulation results.

As shown in Figure 4, we simulated a model implemented of mobile ad hoc network with regard to the assumptions described in Table 2. In this simulation, moving nodes is according to moving model of random track point in which each node moves to a location randomly selected with a specified speed. Then stops are performed for a pause time configured before selecting another random location and repeating the same simulation process. Moving patterns are for six different pause time: 0, 20, 40, 60, 80 and 100 seconds. The results of this simulation shown in figures 5 to 8 by comparing the proposed model, IAOMDV-F, AODVFART and FLM-AODV protocols. 


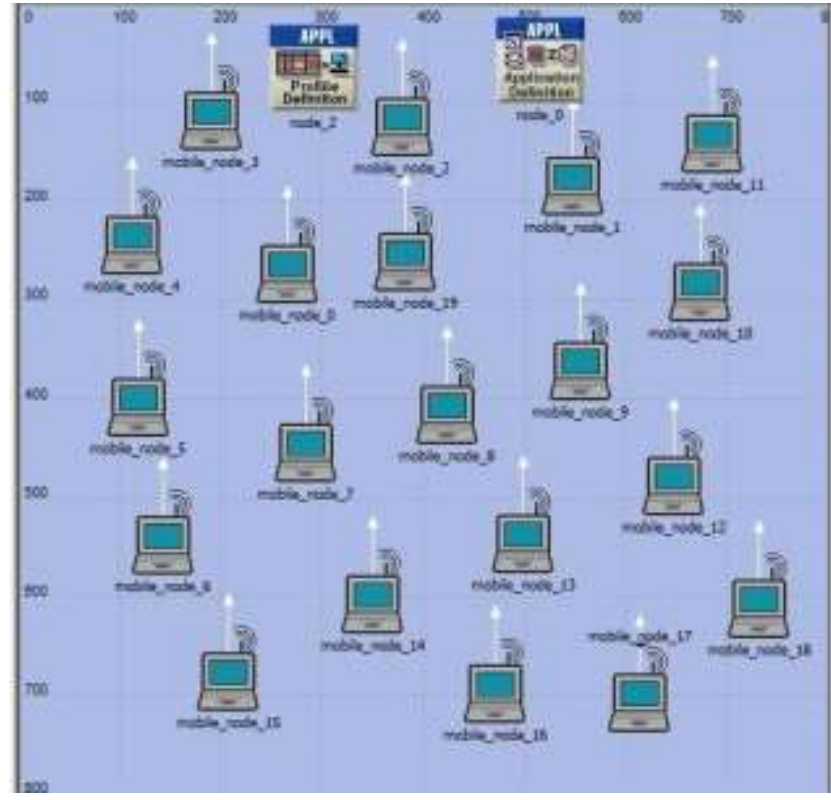

Figure 4. A sample topology of MANET

Table 2. Simulation Parameters

\begin{tabular}{cc}
\hline Parameters & Values \\
\hline Number of mobile nodes & 20 \\
Simulation area & $800 \mathrm{~m}^{*} 800 \mathrm{~m}$ \\
Simulation time & $100 \mathrm{~s}$ \\
Pause time for mobile nodes & $20 \mathrm{~s}$ \\
Maximum speed for mobile nodes & $0-12 \mathrm{~m} / \mathrm{s}$ \\
Packet size & 512 bytes \\
Traffic type & CBR \\
Transmission range for mobile nodes & $150 \mathrm{~m}$ \\
\hline
\end{tabular}

In this simulation, compare the three protocols been done based on the following indexes:

a. Network Throughput: it is the maximum rate that the network equipment can bear in the case of no data loss.

b. Network Delay: it includes the routing discovery delay, waiting delay of packets in the interface queues, transmission delay and retransmission delay of the MAC layer.

c. Routing Discovery Time: it is the time from launching the routing discovery of the source node to receiving the RREP packet and establishing the route.

d. Average Number of Hops per Route: it is defined as the average time of the total number of hops passed by the packet sent from the source node to the destination node, in the whole simulation process.

Figure 5 shows the average performance in terms of throughput for different pause times in a network of 20 nodes. The X-axis represents the pause times and the $\mathrm{Y}$-axis represents the throughput. The simulation in Figure 5 shows that throughput of the proposed method with regard to the parameters considered in fuzzy logic system is better than AODVFART, IAOMDV and FLM-AODV protocols. The reason is that in the proposed method it is used of the two most important parameters of energy and the node degree. The parameter of node degree is tries to select the nodes that are near each other which lead to low power consumption to transmit and receive data from other nodes and thus will affect the network throughput. 


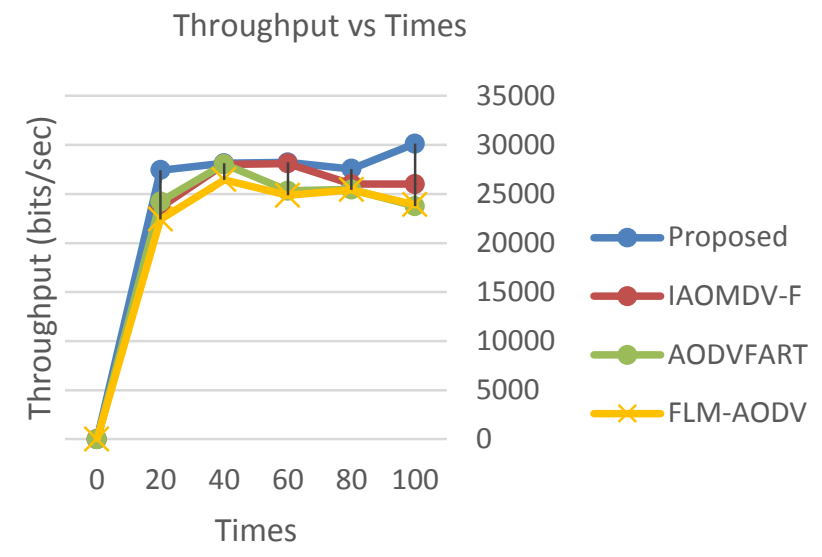

Figure 5. Throughput at different pause times for 20 nodes

Figure 6 shows network delay of the proposed method, AODVFART, IAOMDV-F and FLM-AODV protocols for different pause times. The $X$-axis represents the pause times and the Y-axis represents the network delay. From Figure 6 , it is observed that the average network delay of the proposed method is lesser than other protocols. The reason is that one of the factors implicated in network delay is route discovery delay. So the proposed method has the better route discovery time because close nodes have faster sending time and less sending delay. As a result, the amount of network delay will be more efficient than AODVFART, IAOMDV-F and FLM-AODV protocols.

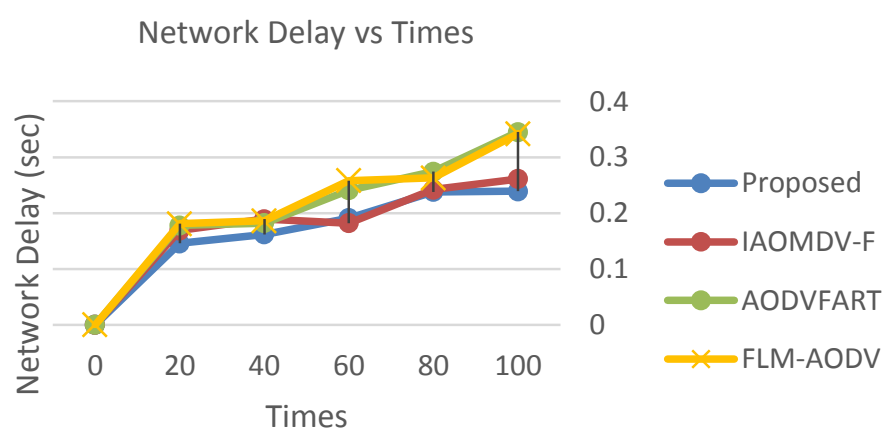

Figure 6. Delay at different pause time for 20 nodes

Figure 7 shows the route discovery time by all four protocols. The $X$-axis represents the pause times and the $\mathrm{Y}$-axis represents the route discovery time. Figure 7 shows that the proposed model reduces route discovery time. The reason is that in the proposed method, nodes that are close together are selected as nodes along the route and in case of failure of a link, it spends less time to discover a new route. As a result, the proposed method will have less discovery route time than AODVFART, IAOMDV-F and FLM-AODV protocols. 


\section{Route Discovery time vs Times}

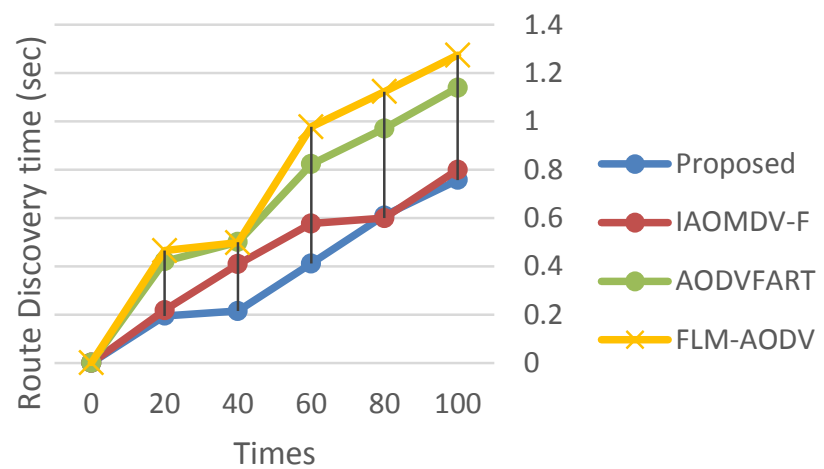

Figure 7. Route discovery time at different pause time for 20 nodes

Figure 8 shows the number of hops the proposed method compared to AODVFART, IAOMDV-F and FLM-AODV protocols for different pause times. The $X$-axis represents the pause time and the $\mathrm{Y}$-axis represents the number of hops. Figure 10 shows that the number of hops the proposed method is less than AODVFART, IAOMDV-F and FLM-AODV protocols. The reason is to choose the shortest route in the proposed method. To do this, some nodes can be chosen as the nodes of route which have a short distance towards each other.

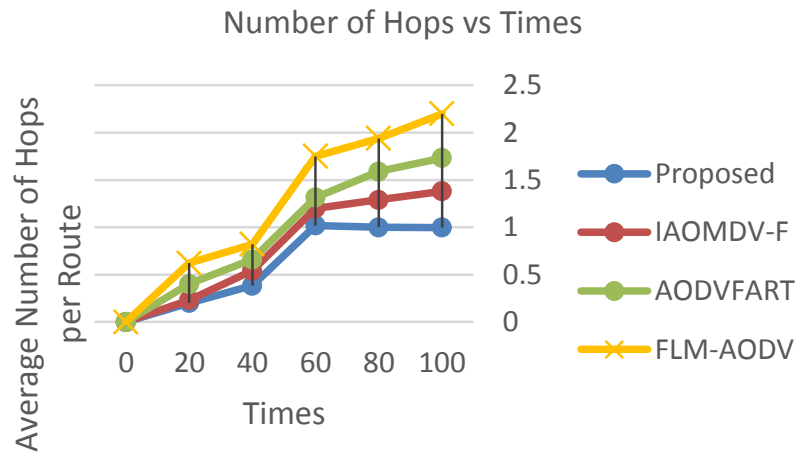

Figure 8. Hop count at different pause time for 20 nodes

\section{Conclusion}

In this paper, we used fuzzy logic to improve routing protocol on demand. The reason for using fuzzy logic is uncertainties in the values of the effective parameters in selecting the optimal route, particularly for mobile ad hoc networks. In the proposed method, we used the four effective parameters in selecting the route including battery energy, node degree, node mobility and bandwidth as input parameters of fuzzy logic system. With the help of the rules base table, we computed the amount of stability of links for each of the candidate routes. Finally, among the candidate routes, we selected the path that has more stability links than any other path send information from the source node to the destination node. In order to evaluate the proposed method, we compared it with AODVFART, IAOMDV-F and FLM-AODV methods by taking the same conditions simulated. Simulation results show that the proposed model outperforms IAOMDV-F, AODVFART and FLM-AODV methods, especially in a high mobility environment. 


\section{References}

[1] Gopi AP, Babu ES, Raju CN, Kumar SA. Designing an adversarial model against reactive and proactive routing protocols in Manets: A comparative performance study. International Journal of Electrical and Computer Engineering (IJECE). 2015 1; 5(5):1111-1118.

[2] $\mathrm{Vu}$ TK, Kwon S. Mobility-assisted on-demand routing algorithm for MANETs in the presence of location errors. The Scientific World Journal. 2014; 18; 1-11.

[3] Sivakumar B, Bhalaji N, Sivakumar D. A survey on investigating the need for intelligent power-aware load balanced routing protocols for handling critical links in MANETs. The Scientific World Journal. $2014 ; 23 ; 1-11$

[4] Alaa A, Najlae I. Mobile Ad hoc Networks: Modelling, Application and Data Routing Evaluation. International Journal of Informatics and Communication Technology (IJ-ICT). 2017; 22; 6(1):20-30.

[5] Jain B, Shrivastava L, Bhadauria SS. Performance Analysis of AODV with Fuzzy based Active Route Timeout Interval in MANET. Journal of Basic and Applied Engineering Research. 2015; 2(16):13881393.

[6] Agarwal MM, Jhankal AK, Govil MC, Sinha M. Fuzzy Logic Controlled Energy Efficient Routing in Mobile Ad-Hoc Networks. International Journal of Computer Networks and Wireless Communications. 2015; 5(4):556-561.

[7] Sharma V, Alam B, Doja M.N. Comparative Study of Fuzzy Logic Mobility Based FLM-AODV Routing Protocol and AODV in MANETs. Indonesian Journal of Electrical Engineering and Computer Science (IJEECS). 2017; 7(1):158-163.

[8] Mendel JM. Uncertain rule-based fuzzy logic systems: introduction and new directions. Upper Saddle River: Prentice Hall PTR; Jan 2001.

[9] Chettibi S, Chikhi S. Dynamic fuzzy logic and reinforcement learning for adaptive energy efficient routing in mobile ad-hoc networks. Applied Soft Computing. 2016; 38:321-8.

[10] AAbbas NI, Ilkan M, Ozen E. Fuzzy approach to improving route stability of the AODV routing protocol. EURASIP Journal on Wireless Communications and Networking. 2015; 1: 235.

[11] Prasanthi VV, Setty SP. Enhancing the Performance of AODV in MANETs using Fuzzy logic. International Journal of Computer Science and Information Technologies. 2015; 6(5):4748-4750.

[12] BBaskaran A, Chaudhari N, Caytiles RD, lyengar NC. Fuzzy Optimized and Bee inspired Routing Protocol for Improved QoS in Mobile Ad Hoc Networks. International Journal of Control and Automation. 2016; 9(8):391-402.

[13] TTabatabaei S, Teshnehlab M, Mirabedini SJ. Fuzzy-based routing protocol to increase throughput in mobile ad hoc networks. Wireless Personal Communications. 2015; 84(4):2307-25.

[14] NNaruephiphat W, Usaha W. Balanced energy-efficient routing in MANETs using reinforcement learning. InInformation Networking, 2008. ICOIN 2008. International Conference on 2008 Jan 23. IEEE. 2008; 1-5.

[15] SSu BL, Wang MS, Huang YM. Fuzzy Logic Weighted Multi-Criteria of Dynamic Route Lifetime for Reliable Multicast Routing in Ad Hoc Networks. Expert Systems with Applications. 2008; 35(1):47684. 